\title{
Simulation of advanced ultrasound systems using Field II
}

\author{
Jensen, Jørgen Arendt
}

Published in:

IEEE International Symposium on Biomedical Engineering 2004

Link to article, DOI:

10.1109/ISBI.2004.1398618

Publication date:

2004

Document Version

Publisher's PDF, also known as Version of record

Link back to DTU Orbit

Citation (APA):

Jensen, J. A. (2004). Simulation of advanced ultrasound systems using Field II. In IEEE International Symposium on Biomedical Engineering 2004 (pp. 636-639). IEEE. https://doi.org/10.1109/ISBI.2004.1398618

\section{General rights}

Copyright and moral rights for the publications made accessible in the public portal are retained by the authors and/or other copyright owners and it is a condition of accessing publications that users recognise and abide by the legal requirements associated with these rights.

- Users may download and print one copy of any publication from the public portal for the purpose of private study or research.

- You may not further distribute the material or use it for any profit-making activity or commercial gain

- You may freely distribute the URL identifying the publication in the public portal

If you believe that this document breaches copyright please contact us providing details, and we will remove access to the work immediately and investigate your claim. 


\title{
SIMULATION OF ADVANCED ULTRASOUND SYSTEMS USING FIELD II
}

\author{
Jørgen Arendt Jensen
}

\author{
Center for Fast Ultrasound Imaging, Ørsted•DTU, Bldg. 348, \\ Technical University of Denmark, DK-2800 Kgs. Lyngby, Denmark
}

\begin{abstract}
The background and basic features of the Field II simulation program are described. It can simulate any linear ultrasound system, which can use single or multi-element transducers. Any kind of apodization, focusing, and excitation can be used. The basic theory behind the program's use of spatial impulse responses is explained. A simulation example for a synthetic aperture spread spectrum flow systems is described. It is shown how the advanced coded excitation can be set up, and how the simulation can be parallelized to reduce the simulation time from 17 months to 391 hours using a 32 CPU Linux cluster.
\end{abstract}

\section{INTRODUCTION}

Simulation has played a major role in all engineering disciplines. In electrical engineering SPICE has been used for accurate simulation of linear and non-linear electrical circuits. In mechanical and civil engineering finite element and finite difference programs are standard tools for evaluating the performance and reliability of mechanical structures, materials, and buildings. Often very accurate results can be obtained, which can save many iterations in prototype development.

The use of simulation is less wide-spread in medical imaging, since the volume of products is smaller and the field has been more experimentally based. This has started to change in the last decade with the progress in computer power, and more tools have become available, that realistically can model the complex interaction between systems and the human body in medical imaging.

This paper will describe our efforts within simulation of medical ultrasound systems. The first program was written in 1990 and initial results published in [1]. The program was re-written in 1995 to be more general with an interface to Matlab for signal processing [2]. This paper will give an overview of the theory behind the program and its features, and a new complex example of spread spectrum synthetic aperture ultrasound flow imaging is presented to illustrate the program's capabilities.

This work was supported by grant 9700883,9700563 and 26-01-0178 from the Danish Science Foundation and by B-K Medical A/S, Denmark.

\section{SPATIAL IMPULSE RESPONSES}

The Field II program is based on calculation of spatial impulse responses as suggested by Tupholme [3] and Stepanishen [4]. The concept is analogues to impulse responses in any linear system, with the notable difference that the impulse responses are dependent on the relative position between the transmitter and the receiver, hence the term spatial impulse response. The approach assumes a homogeneous bounded medium, where the pressure is sufficiently small to ensure linear wave propagation. Hereby both single element and multi-element arrays can be handled, since the response is a mere superposition of the responses from the different elements properly phase aligned.

The method can be described using Huygens' principle, where the impulse response is calculated from a summation of all spherical waves from the aperture area $S$ as:

$$
h\left(\vec{r}_{1}, t\right)=\int_{S} \frac{\delta\left(t-\frac{\left|\vec{r}_{1}-\vec{r}_{2}\right|}{c}\right)}{2 \pi\left|\vec{r}_{1}-\vec{r}_{2}\right|} d S,
$$

where $\left|\vec{r}_{1}-\vec{r}_{2}\right|$ is the distance from the transducer at position $\vec{r}_{2}$ to the field point at $\vec{r}_{1}, \delta(t)$ is the Dirac delta function, and $c$ is the speed of sound.

The calculation can be done analytically for a number of apertures like the round piston [3], for a circular convex element [5], and for a rectangle element [6]. Closed form solutions can, however, not be found for all types. Especially the introduction of $e . g$. double curved geometries or apodization over the transducer surface leads to analytically unsolvable integrals. Field II therefore divides the aperture into smaller mathematical elements to describe advanced shapes. Apodization is handled by assigning different weights for different elements. The most frequently used mathematical element is the rectangle, where the farfield response is used [1], which makes the calculation very fast even for many elements [7]. The program can also use triangles [8] or bounding lines [9], where the exact spatial impulse response is calculated.

Spatial impulse responses are often very short for small array elements, which makes them difficult to calculate accurately with a low sampling frequency. An integration of the responses and an analog time scale is therefore used in 
the program to capture all the energy of the responses. This has been efficient in lowering the sampling frequency by a factor of 10 to 20 [7].

Homogeneous attenuation can also be handled by the program. It is introduced as a minimum phase filter acting on the spatial impulse response [10].

Any kind of linear ultrasound field can be calculated using spatial impulse responses. The emitted pressure field $p\left(\vec{r}_{1}, t\right)$ is given by:

$$
p\left(\vec{r}_{1}, t\right)=\rho_{0} \frac{\partial v(t)}{\partial t} * h\left(\vec{r}_{1}, t\right),
$$

where $\rho_{0}$ is the density of the medium and $\partial v(t) / \partial t$ is the acceleration of the front face of the transducer. The convolution model ensures that any kind of excitation can be handled by the program. To separate excitation and the transducer's electro-mechanical impulse response, two convolutions are performed as the excitation is convolved onto (2).

The received voltage signal for the pulse echo field is [11]:

$$
\begin{aligned}
v_{r}\left(\vec{r}_{1}, t\right) & =v_{p e}(t) * f_{m}\left(\vec{r}_{1}\right) * h_{p e}\left(\vec{r}_{1}, t\right) \\
f_{m}\left(\vec{r}_{1}\right) & =\frac{\Delta \rho\left(\vec{r}_{1}\right)}{\rho_{0}}-\frac{2 \Delta c\left(\vec{r}_{1}\right)}{c},
\end{aligned}
$$

where the scattering signal $f_{m}\left(\vec{r}_{1}\right)$ arises from spatial variations in density $\Delta \rho\left(\vec{r}_{1}\right)$ and speed of sound $\Delta c\left(\vec{r}_{1}\right)$. Here $h_{p e}\left(\vec{r}_{1}, t\right)$ is the two-way spatial impulse response, that is a convolution between the impulse response of the transmitting and receiving aperture. The impulse response $v_{p e}(t)$ includes the excitation convolved with both the transducers electro-mechanical impulse response in transmit and receive. The final signal for a collection of scatterers is calculated as a linear sum over all signals from the different scatterers. This can also be viewed as a spatial convolution between the pulse-echo impulse response and the scatterers.

The continuous wave fields are found from a Fourier transform of the emitted field $\mathcal{F}\left\{p\left(\vec{r}_{1}, t\right)\right\}$ and the pulseecho field $\mathcal{F}\left\{v_{r}\left(\vec{r}_{1}, t\right)\right\}$ for any frequency. All fields can, thus, be derived from the spatial impulse response.

\section{FROM POINT SPREAD FUNCTIONS TO ADVANCED IMAGING}

The first version of the Field simulation system was developed in 1990, and is described in [1]. It was developed on an Apollo DN3000 workstation with a Linpack performance of 72 kflops. The system was entirely written in $\mathrm{C}$ with a simple menu interface and was geared to simulate simple point spread functions with a fixed transducer, apodization and focusing. An accurate point spread function could be obtained in minutes as shown in Fig. 1. The pulse-echo response from a fine needle was obtained as a function of time
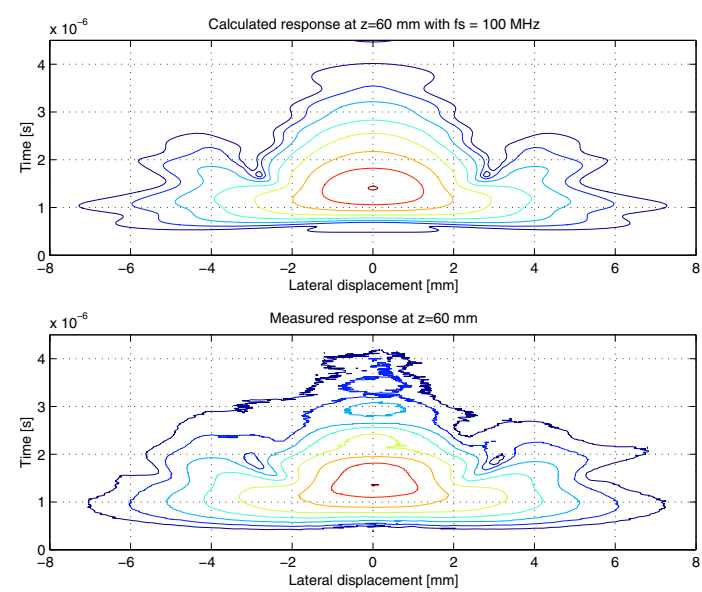

Fig. 1. Point spread function for a concave, focused transducer. Top: simulation, bottom: tank measurement ( $6 \mathrm{~dB}$ between the contour lines)

and lateral position. An $8.1 \mathrm{~mm}$ radius convex transducer focused at $150 \mathrm{~mm}$ was probed at $60 \mathrm{~mm}$ from the transducer surface. An accuracy down to fractions of a percent could be attained.

The initial version of the program could simulated RF signals for a collection of scatterers. The dynamic focusing and apodization capabilities were, however, limited, and it was very difficult to include the other signal processing involved in ultrasound imaging into the program without writing large amounts of code. This would also be inflexible in terms of exploring newer imaging methods.

It was therefore decided to make a version which was based on a language structure rather than a menu structure. This, however, complicates the program writing and would have a large overhead in terms of compilation and debugging. A partial solution was therefore adopted in which the core was written in $\mathrm{C}$, and the calling of the program, and subsequent signal processing, was handled in Matlab. This makes the simulation fast as it is performed in C, and flexibility is still maintained through the general processing capabilities of Matlab. This version was described in [2]. The capabilities of the new version are listed below:

- Transducer modeled by dividing it into rectangles, triangles and bounding lines.

- C program interfaced to Matlab.

- Can handle any transducer geometry. Current types: piston and concave single element, linear, phased, and convex arrays, 2D matrix, elevation focused array.

- Any focusing, apodization, and excitation pulse.

- Can calculate all types of fields (emitted, received, pulsed, CW).

- Signals from all elements or the signal combined after focusing can be calculated. 
- Can generate artificial ultrasound images (phased, linear, and convex array images, anatomic and flow images with multiple receive and transmit foci, and synthetic aperture images).

- Versions for: Windows, Linux, HP, MAC OS X, SUN, SGI, DEC Station (ALPHA), IBM AIX.

- The program can be downloaded from: http://www.es.oersted.dtu.dk/staff/jaj/field/

Currently the program is only maintained by the author to ensure consistency of the code and accuracy of the calculated responses. Older versions are kept as source files and the use of a source control systems (CVS) are currently being investigated. Support to the users are given through a rudimentary users' guide, Matlab help files, and several examples of use on the programs web-site listed above.

\section{SIMULATION OF SPREAD SPECTRUM SYNTHETIC APERTURE FLOW IMAGING}

To illustrate the program's possibilities, a synthetic aperture (SA) flow system has been simulated. In a SA system a number of emissions are made with different elements, and the received signals from all receiving elements are combined to focus a high resolution image in the full region of interest. The challenge in SA flow imaging is to make a limited number of emissions to maintain high correlation between images and still attain a high resolution, signalto-noise ratio, and image contrast. This can be solved using spread spectrum techniques. It was introduced in [12], which demonstrated the use for B-mode imaging.

In this paper a new approach of using spread spectrum emission for flow imaging is introduced. The estimation scheme is based on the SA flow estimation method detailed in [13]. The available transducer bandwidth is divided into 32 bands consisting of 2 sets of 16 bands each. The bands in each set are mutually orthogonal, since they do not overlap in the frequency domain. The combination of the two sets covers the full transducer bandwidth, and the combination therefore attains the resolution of a normal pulse excitation. The advantage is that all 16 bands can be emitted simultaneously, so that the full aperture can be used in emission. Both a high resolution and a large signal-to-noise ratio can potentially be obtained by this method. Further, a focused transmission with an F-number of 1/2 is used, and the virtual source point is placed in front of the transducer. This increases both signal-to-noise ratio and the localization of the center of this virtual source, when many elements are used for transmission.

An example of the emitted field is shown in Fig. 2, where the emitted pressure at the emission focus has been calculated for the two emissions. The frequency bands are assigned from low to high frequencies from left to right in the plot. The largest signal is seen around the center of the
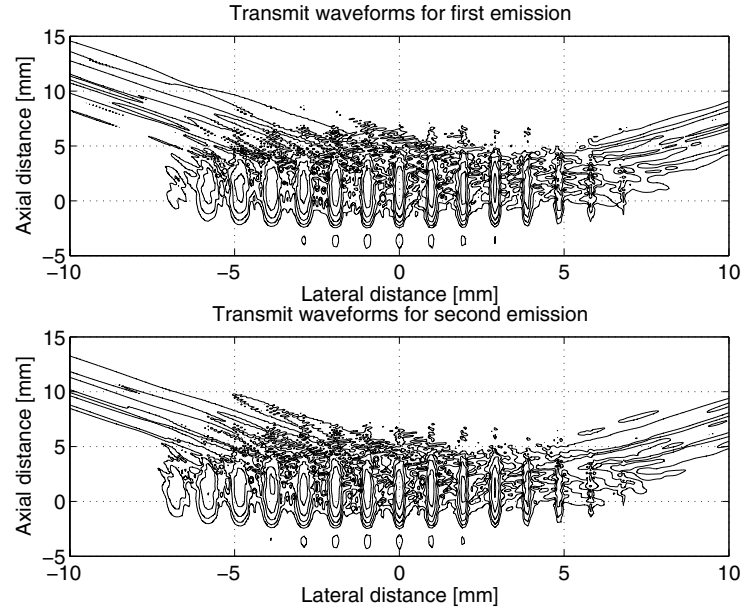

Fig. 2. Emitted waveforms for the two emissions. There is $6 \mathrm{~dB}$ between the contours in the plots.

array, since the frequency band here is closets to the transducer center frequency. A $7 \mathrm{MHz}$ phased array transducer with $\lambda / 2$ pitch and an elevation focus at $36.5 \mathrm{~mm}$ was used.

The simulation is performed by defining two transmitting apertures. For each of the transmitting virtual points a special excitation waveform is assigned for the transmitting elements. Normally 8 elements can be used for transmission per band, since there are 16 bands and a 128 element transducer. A Hanning apodization is, however, applied onto the elements, so that the transmitting elements for two virtual sources can overlap and 16 elements used for transmission for each source. The combined amplitude of the two waveforms is hereby close to one, and the aperture therefore both uses all elements and the full amplitude of them simultaneously.

The approach necessitates the setting of individual signals on individual elements and the sub-sample delays are introduced by manipulation of the phase in the waveforms.

\section{PROCESSING AND RESULTS}

The program simulates the signals received by all 128 transducer elements, and a matrix with signals results for each pulse-echo simulation. The separation of the received signal from each of the virtual sources is done by applying a matched filter for each band on all the received signals. The result is the received signal on a specific element for one of the bands. The matched filter also equalizes the phase of the signals, so they all become zero phase signals. The signals are then focused by adding them for all elements, all bands and both emissions with a compensation for the travel time from the virtual source to the receiving element.

The flow is simulated for a random collection of point scatterers in a tube with a laminar, parabolic flow with a 


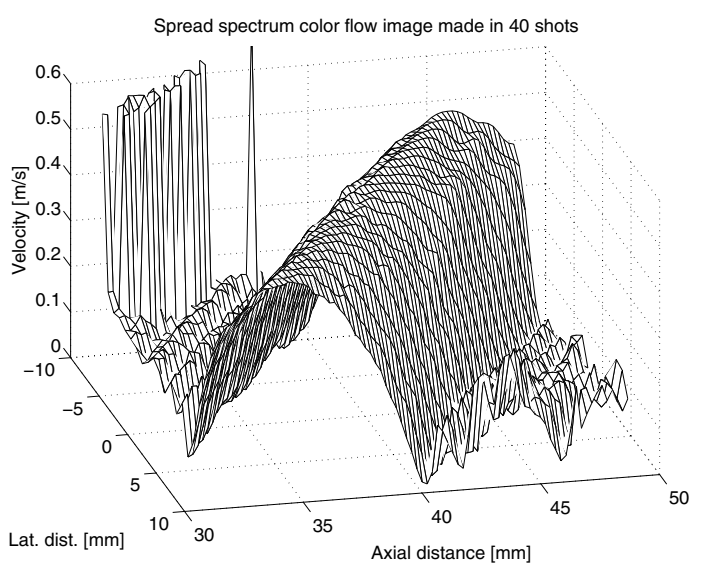

Fig. 3. Spread spectrum flow image made in 40 emissions.

peak velocity of $0.5 \mathrm{~m} / \mathrm{s}$. The flow angle was 60 degrees and 31,500 point scatterers were used. The scatterers are propagated between pulse emissions, and the pulse-echo response is calculated for 2000 emissions. The full simulation was performed on a $32 \mathrm{CPU}$ Linux cluster under Matlab 6.5. It took 391 hours to complete equal to 12,512 hours of simulation time (corresponding to roughly 17 months of CPU time). All computers have access to the same disk space using NFS (Network File System), and the parallelization was done by splitting the job into files, with one file for one pulse emission. The computer then pre-stored the result file to reserve part of the job. The method has worked well for simulations that takes from minutes to hours for the individual jobs for more then 50 CPUs running Linux.

The result of the simulation is shown in Fig. 3. The velocity has been determined by estimating the velocity using a cross-correlation approach. The received data is focused along the flow direction as in [13], and the signals are then cross-correlated to find the displacement between pulse emissions. Dividing with the time between emissions then yields the velocity. The full velocity image has been made in only 40 emissions, where traditional imaging would demand roughly 800 emissions. The parabolic shape of the flow is estimated. Erroneous peaks are seen outside the vessel, where there is no signal from the scatterers.

\section{CONCLUSION}

The background for the Field II program has been described along with an advanced example of its use. The program has now been in use for more than 10 years, and its has demonstrated that accurate results are obtained. The program is free to download for a number of different platforms, and more than 60 journal articles has been published from its use by a large number of research groups (Science Citation Index search, February 2004).

\section{REFERENCES}

[1] J. A. Jensen and N. B. Svendsen, "Calculation of pressure fields from arbitrarily shaped, apodized, and excited ultrasound transducers," IEEE Trans. Ultrason., Ferroelec., Freq. Contr., vol. 39, pp. 262-267, 1992.

[2] J. A. Jensen, "Field: A program for simulating ultrasound systems," Med. Biol. Eng. Comp., vol. 10th Nordic-Baltic Conference on Biomedical Imaging, Vol. 4, Supplement 1, Part 1, pp. 351-353, 1996b.

[3] G. E. Tupholme, "Generation of acoustic pulses by baffled plane pistons," Mathematika, vol. 16, pp. 209224, 1969.

[4] P. R. Stepanishen, "Transient radiation from pistons in an infinite planar baffle," J. Acoust. Soc. Am., vol. 49, pp. 1629-1638, 1971.

[5] A. Penttinen and M. Luukkala, "The impulse response and nearfield of a curved ultrasonic radiator," J. Phys. D: Appl. Phys., vol. 9, pp. 1547-1557, 1976.

[6] J. C. Lockwood and J. G. Willette, "High-speed method for computing the exact solution for the pressure variations in the nearfield of a baffled piston," $J$. Acoust. Soc. Am., vol. 53, pp. 735-741, 1973.

[7] J. A. Jensen, "Speed-accuracy trade-off in computing spatial impulse responses for simulating medcial ultrasound imaging," J. of Computational Acoustics, vol. 9, no. 3, pp. 731-744, 2001.

[8] J. A. Jensen, "Ultrasound fields from triangular apertures," J. Acoust. Soc. Am., vol. 100(4), pp. 20492056, 1996a.

[9] J. A. Jensen, "A new calculation procedure for spatial impulse responses in ultrasound," J. Acoust. Soc. Am., vol. 105, no. 6, pp. 3266-3274, 1999.

[10] J. A. Jensen, D. Gandhi, and W. D. O'Brien, "Ultrasound fields in an attenuating medium," in Proc. IEEE Ultrason. Symp., 1993, pp. 943-946.

[11] J. A. Jensen, "A model for the propagation and scattering of ultrasound in tissue," J. Acoust. Soc. Am., vol. 89, pp. 182-191, 1991a.

[12] F. Gran and J. A. Jensen, "Multi element synthetic aperture transmission using a frequency division approach," in Proc. IEEE Ultrason. Symp., 2003, pp. 1942-1946.

[13] J. A. Jensen and S. I. Nikolov, "Transverse flow imaging using synthetic aperture directional beamforming," in Proc. IEEE Ultrason. Symp., 2002, pp. 1488-1492. 